\title{
Late-Phase Observations of a Super-Chandrasekhar SN Ia
}

\author{
M. Yamanaka ${ }^{1}$, K. S. Kawabata ${ }^{1}$, K. Maeda ${ }^{2}$, M. Tanaka ${ }^{2}$, \\ M. Yoshida ${ }^{1}$, T. Hattori ${ }^{3}$, K. Nomoto ${ }^{2}$, T. Komatsu ${ }^{4}$, and \\ T. Okushima ${ }^{4}$ \\ ${ }^{1}$ Hiroshima Astrophysical Science Center, Hiroshima University, \\ Higashi-Hiroshima, Hiroshima 739-8526, Japan \\ email: myamanaka@hiroshima-u.ac.jp \\ ${ }^{2}$ Institute for the Physics and Mathematics of the Universe, \\ University of Tokyo, Kashiwa, Japan \\ ${ }^{3}$ Subaru Telescope, National Astronomical Observatory of Japan, \\ Hilo, HI, USA \\ ${ }^{4}$ Department of Physical Science, Hiroshima University, \\ Kagamiyama 1-3-1, Higashi-Hiroshima 739-8526, Japan
}

\begin{abstract}
A super-Chandrasekhar (SC) supernova (SN) has an extremely high luminosity and a slow decline rate of the light curve in the early-phase. We present late-phase observations of the SC SN 2009dc. We find that the optical luminosity a year after maximum is much fainter than that expected from its early luminosity. We attempt to fit the analytic light curve model to the observations using Arnett's rule. The model successfully explains the light curves until 120 days. This suggests that the extremely high luminosity originates from the ${ }^{56} \mathrm{Ni}$ decay. We suggest that the late-phase decline would be caused by dust formation. The existence of strong carbon features in early-phase spectra would support this scenario. We also find a blend of [Ca II] and $[\mathrm{Ni} \mathrm{II}]$ in its late-phase spectrum. This indicates that the calcium is distributed in the inner layer along with nickel and iron. We conclude that the mixing may occur in the inner parts of the ejecta.
\end{abstract}

Keywords. supernovae: individual (SN 2009dc)

\section{Introduction}

SN 2009dc exhibits an extremely high luminosity $\left(\mathrm{M}_{V}=-20.3\right)$ and a very slow decline rate $\left(\Delta \mathrm{m}_{15}(B)=0.65\right)$ in its early-phase (Yamanaka et al. 2009, Silverman et al. 2011, Taubenberger et al. 2011). Spectra show the deep C II $\lambda 6580$ absorption line, which is not seen in typical SNe Ia. The line velocity of Si II and C II is estimated to be 8,000 $\mathrm{km} \mathrm{s}^{-1}$, which is slower than the $12,000 \mathrm{~km} \mathrm{~s}^{-1}$ of typical SNe Ia. The observational properties of SN 2009dc are similar to those of SN 2006gz. The ${ }^{56} \mathrm{Ni}$ mass is calculated to be $1.8 \mathrm{M}_{\odot}$ from the peak quasi-bolometric luminosity, assuming that the extinction is $A_{V}=0.43$ and the rise time is 20 days (Yamanaka et al. 2009). The presence of large amounts of carbon supports that the total ejected mass is even more. Low polarization of the continuum also support this (Tanaka et al. 2010). From these observational facts, Yamanaka et al. 2009 conclude that SN 2009dc is a super-Chandrasekhar SN Ia (see also Silverman et al. 2011, Taubenberger et al. 2011).

\section{Late-Phase Observations}

A late-phase spectrum is a useful tool to understand the inner structure of SN ejecta. As an SN expands, the ejecta become optically thin. Thus, late-phase observations of an 
$\mathrm{SN}$ inform us of the inner structure of the ejecta. We performed optical photometric and spectroscopic observations using the $8.2 \mathrm{~m}$ Subaru telescope with FOCAS (Kashikawa et al. 2002) on May 13, 2010 (383 days after $B$-band maximum). For photometry, we used $B, V, R_{\mathrm{C}}$ and $I_{\mathrm{C}}$ filters. For spectroscopy, the spectral coverage is $3800-10200 \AA$ and the resolution is $R \sim 500$.

\section{Results}

We constructed the quasi-bolometric light curves using $B, V, R_{\mathrm{C}}$ and $I_{\mathrm{C}}$-band light curves (Figure 1). The early-phase light curves are from Yamanaka et al. 2009. The quasi-bolometric light curves indicate that the late-phase luminosity is fainter than that expected from the early-phase luminosity. We attempt to fit to the light curve using Arnett's rule (Arnett et al. 1982). The analytic model successfully explains the light curves until 120 days after $B$-band maximum. Thus, we conclude that the early-phase luminosity originates from ${ }^{56} \mathrm{Ni}$ decay. We suggest that the late-phase luminosity is decreased by dust formation. The presence of carbon supports this scenario. The much redder color than a typical SN Ia at late-phase also supports it. In Figure 2, we consider the possible identification of [Ca II] $\lambda 7299$ in the late-phase spectrum, by comparing the spectrum of SN 2009dc with a typical SN Ia, SC SN 2006gz (Maeda et al. 2009) and with a corecollapse SN 1993J. This calcium line shows a redshift of $300 \mathrm{~km} \mathrm{~s}^{-1}$, while the nickel and iron emission lines are blueshifted by $600 \mathrm{~km} \mathrm{~s}^{-1}$. This indicates that the calcium is distributed in a layer interior to that of the iron-group elements. This situation could be produced by the mixing in the ejecta.

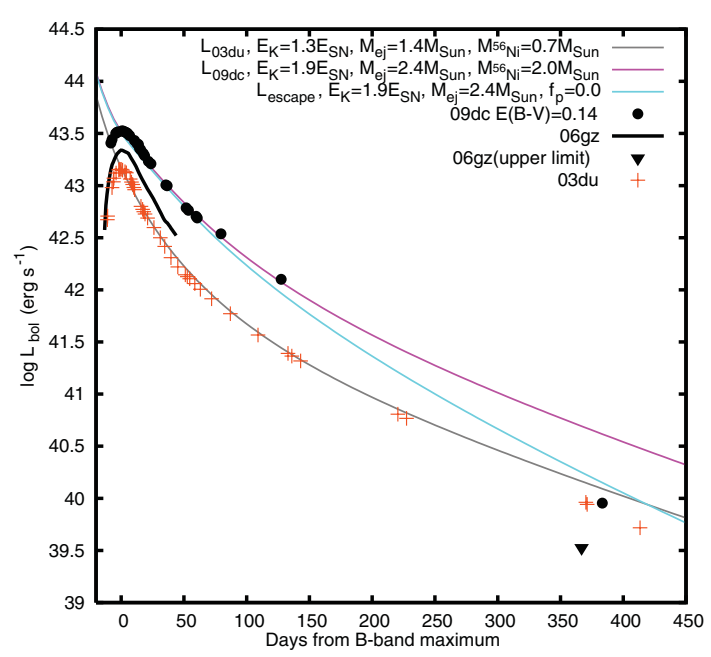

Figure 1. The quasi-bolometric light curve of SN 2009dc compared with those of a typical SN Ia 20003du and another SC SN 2006gz. Analytic light curves are constructed based on Arnett's rule (Arnett et al. 1982) for the total ejected mass of $2.4 \mathrm{M}_{\odot}$ and kinetic energy of $1.9 \times 10^{51} \mathrm{erg}$, denoted as the pink line.

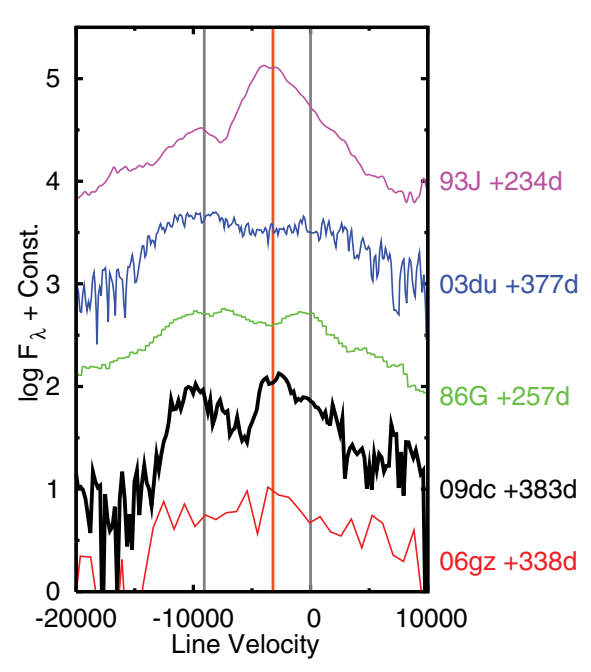

Figure 2. Emission lines of SC SN 2009dc compared with another SC SN 2006gz (Maeda et al. 2009), typical SN Ia $2003 \mathrm{du}$ (Stanishev et al. 2007), subluminous SN 1986G and core-collapse Type IIb SN 1993J. The two vertical gray lines denote the rest frame velocity of $[\mathrm{Fe} \mathrm{II}] \lambda 7155$ and $[\mathrm{Ni} \mathrm{II}] \lambda 7379$. On the other hand, the vertical red line denotes that of $[\mathrm{Ca} \mathrm{II}] \lambda 7299$. 


\section{References}

Arnett, W. D. 1982, ApJ, 253, 785

Kashikawa, N., et al. 2002, PASJ, 54, 819

Maeda, K., et al. 2009, ApJ, 690, 1745

Silverman, J. M., Ganeshalingam, M., Li, W., Filippenko, A. V., Miller, A. A., \& Poznanski, D. 2011, MNRAS, 410, 585

Stanishev, V., et al. 2007, A\&A, 469, 645

Tanaka, M., et al. 2010, ApJ, 714, 1209

Taubenberger, S., et al. 2011, MNRAS, 61

Yamanaka, M., et al. 2009, ApJ, 707, L118 